\title{
Analytical angular solutions for the atom-diatom interaction potential in a basis set of products of two spherical harmonics: two approaches
}

\author{
Mariusz Pawlak $^{1}$ (D) Marcin Stachowiak ${ }^{1}$
}

Received: 29 January 2021 / Accepted: 16 August 2021 / Published online: 25 August 2021

(c) The Author(s) 2021

\begin{abstract}
We present general analytical expressions for the matrix elements of the atom-diatom interaction potential, expanded in terms of Legendre polynomials, in a basis set of products of two spherical harmonics, especially significant to the recently developed adiabatic variational theory for cold molecular collision experiments [J. Chem. Phys. 143, 074114 (2015); J. Phys. Chem. A 121, 2194 (2017)]. We used two approaches in our studies. The first involves the evaluation of the integral containing trigonometric functions with arbitrary powers. The second approach is based on the theorem of addition of spherical harmonics.
\end{abstract}

Keywords Analytical solutions · Spherical harmonics · Associated Legendre polynomials $\cdot$ Atom-diatom interaction potential $\cdot$ Matrix elements

\section{Introduction}

Atomic and molecular collisions at subkelvin temperatures are a very sensitive probe of the interaction potential. The group of Narevicius conducted a few years ago the first merged-beam experiments for low-energy collisions of atoms with diatomic molecules [1-3]. These experiments were cutting-edge attainments in cold controlled chemistry [4-6]. Recently, Narevicius with collaborators directly probed the anisotropy in the atom-diatom collisions through orbiting resonances, revealing its strong role in the interaction energy [7]. Therefore, a high level of theory is needed to inspect and elucidate the measurements in the subkelvin regime, where quantum effects dominate.

The potential describing the interaction between an atom in an $\mathrm{S}$ state and a closed-shell diatomic molecule in the Jacobi coordinate system is a function of

Mariusz Pawlak

teomar@chem.umk.pl

1 Faculty of Chemistry, Nicolaus Copernicus University in Toruń, Gagarina 7, 87-100 Toruń,

Poland 
three coordinates $(R, r, \vartheta)$. The orientation of the molecule and the entire complex is characterized by the vectors $\mathbf{r}$ and $\mathbf{R}$, respectively. Consequently, $r$ is the interatomic separation in the molecule, whereas $R$ is the distance from the atom to the center of mass (COM) of the molecule. The third coordinate $\vartheta$ is the angle between $\mathbf{r}$ and $\mathbf{R}$. The potential may be conveniently expanded in Legendre polynomials as follows [8-10]

$$
\begin{aligned}
V(R, r, \vartheta)= & \sum_{\eta} V_{\eta}(R, r) P_{\eta}(\cos \vartheta) \\
= & V_{0}(R, r)+V_{1}(R, r) \cos \vartheta+V_{2}(R, r) \frac{1}{2}\left(3 \cos ^{2} \vartheta-1\right) \\
& +V_{3}(R, r) \frac{1}{2}\left(5 \cos ^{3} \vartheta-3 \cos \vartheta\right) \\
& +V_{4}(R, r) \frac{1}{8}\left(35 \cos ^{4} \vartheta-30 \cos ^{2} \vartheta+3\right)+\ldots,
\end{aligned}
$$

where $V_{\eta}(R, r)$ are radial functions. Note that the terms with $\eta=1,3, \ldots$ vanish for homonuclear molecules because $V(R, r, \vartheta)=V(R, r, \pi-\vartheta)$. When we consider the atom-diatom complex in the laboratory coordinate frame, the angle $\vartheta$ can be expressed in terms of four angles $\theta_{1}, \phi_{1}, \theta_{2}, \phi_{2}[11]$ :

$$
\begin{aligned}
\cos \left[\vartheta\left(\theta_{1}, \phi_{1}, \theta_{2}, \phi_{2}\right)\right]=\frac{\mathbf{R} \cdot \mathbf{r}}{R r}= & \sin \theta_{1} \cos \phi_{1} \sin \theta_{2} \cos \phi_{2} \\
& +\sin \theta_{1} \sin \phi_{1} \sin \theta_{2} \sin \phi_{2}+\cos \theta_{1} \cos \theta_{2} .
\end{aligned}
$$

The angles $\theta_{1}$ and $\phi_{1}$ specify the direction of the atom-COM axis, whereas $\theta_{2}$ and $\phi_{2}$ indicate the orientation of the diatomic bond.

The adiabatic approach in molecular collisions has a long record, going back to Levine [12]. Klemperer and coworkers [8] introduced the Born-Oppenheimer separation of angular and radial motion for calculating the properties of ground-state van der Waals complexes. A similar concept has been adopted by many other authors, e.g., for spectroscopic studies of weakly bound molecular systems [13-18] or molecular scattering calculations [19-21]. In our investigations, we have employed the newly developed adiabatic variational theory (AVT) for cold anisotropic collision experiments $[11,22,23]$ that allows solving the Schrödinger equation, reducing the multidimensional problem to simpler subproblems without losing physical information, and then calculating reaction rate coefficients. It has been successfully applied for excited metastable helium atoms $\left(\mathrm{He}\left(2^{3} \mathrm{~S}\right)\right)$ colliding with rovibrational ground-state hydrogen molecules, achieving a good, or very good, agreement with the experimental data of the Narevicius group [22-25]. The latest work with AVT confirms the observed strong quantum kinetic isotope effect in low-energy collisions between $\mathrm{He}\left(2^{3} \mathrm{~S}\right)$ and hydrogen isotopologues $\left(\mathrm{H}_{2}, \mathrm{HD}, \mathrm{D}_{2}\right)$ and exhibits that the change of the rovibrational state of the molecule can significantly enhance or suppress the reaction process like a quantum switch [26].

According to AVT, we represent the interaction potential, Eq. (1), in a matrix form in a basis set consisting of products of two spherical harmonics, $\left\{Y_{l_{1}, m_{1}}\left(\theta_{1}, \phi_{1}\right) Y_{l_{2}, m_{2}}\left(\theta_{2}, \phi_{2}\right)\right\}$, where $l_{1}, m_{1}, l_{2}, m_{2}$ are referred to as quantum numbers. 
Such a basis set describes the rotations of the entire complex and the molecule. Up to date, AVT has been only used with $\eta \leq 2$ [11, 22-27], where the matrix elements were calculated directly without using sophisticated methods. For $\eta>2$, the matrix elements become much more complicated and difficult to resolve. Therefore, in the present work, we derive general analytical angular solutions for the following matrix elements (in the Dirac notation)

$$
V_{l_{1}, m_{1}, l_{2}, m_{2}}^{l_{1}^{\prime}, m_{1}^{\prime}, l_{2}^{\prime}, m_{2}^{\prime}}=\left\langle\prod_{j=1}^{2} Y_{l_{j}^{\prime}, m_{j}^{\prime}}\left(\theta_{j}, \phi_{j}\right)\left|V\left(R, r, \vartheta\left(\theta_{1}, \phi_{1}, \theta_{2}, \phi_{2}\right)\right)\right| \prod_{j=1}^{2} Y_{l_{j}, m_{j}}\left(\theta_{j}, \phi_{j}\right)\right\rangle .
$$

This is a purely mathematical issue and it boils down to the evaluation of appropriate integrals, which can be further used to investigate thoroughly the effect of anisotropy on the resonant structure in reaction cross sections and rate coefficients of atoms colliding with diatoms. In general, the inclusion of higher terms $(\eta>2)$ of the Legendre polynomials expansion in AVT may lead to a better agreement of the theoretical predictions with the experimental findings and a deeper understanding of quantum phenomena in chemical reactions (as, for instance, Penning ionization, interatomic or intermolecular Coulombic decay, Auger effect), especially at very low temperatures (below 1 kelvin). Moreover, the first detection of $\mathrm{He}\left(2^{3} \mathrm{~S}\right)$ on an exoplanet in 2018 [28] significantly extended the role of theoretical chemistry in astrochemistry. The interaction and collisions of $2^{3} \mathrm{~S}$ helium with ubiquitous molecular hydrogen and other diatomic molecules are expected to be intensively studied in the next years.

The problem might seem to be similar to the one considered by Percival and Seaton in Ref. [29]. The authors found the matrix elements of $P_{\eta}(\cos \vartheta)$ in the following basis set $\left\{\sum_{m_{1}, m_{2}}\left\langle l_{1}, l_{2}, m_{1}, m_{2} \mid L M\right\rangle Y_{l_{1}, m_{1}}\left(\theta_{1}, \phi_{1}\right) Y_{l_{2}, m_{2}}\left(\theta_{2}, \phi_{2}\right)\right\}$, where $\left\langle l_{1}, l_{2}, m_{1}, m_{2} \mid L M\right\rangle$ are the Clebsch-Gordan coefficients. However, they imposed constraints that $\left|l_{1}-l_{2}\right| \leq L \leq l_{1}+l_{2}$ and $M=m_{1}+m_{2}$. This action is physically well-motivated - the total angular momentum $L$ is a rigorously good quantum number. Since then, the other authors have been widely using the solutions of Percival and Seaton. However, the solutions cannot be adopted to the above-mentioned AVT, which is a non-standard method, where $m_{1}$ and $m_{2}$ are input parameters, but not $L$ and $M$. Nevertheless, the studies presented in this work are based on a methodology different from that in Ref. [29] and to our knowledge have not been conducted previously.

\section{Evaluation of the integral involving arbitrary powers of trigonometric functions}

First, we represent the Legendre polynomials in Eq. (1) as follows:

$$
P_{\eta}(\cos \vartheta)=2^{\eta} \sum_{k=0}^{\eta}\left(\begin{array}{l}
\eta \\
k
\end{array}\right)\left(\begin{array}{l}
\alpha \\
\eta
\end{array}\right) \cos ^{k} \vartheta
$$

where $\alpha=\frac{\eta+k-1}{2}$. Note that $\left(\begin{array}{l}\alpha \\ \eta\end{array}\right)$ is the generalized binomial coefficient defined as 


$$
\left(\begin{array}{l}
\alpha \\
\eta
\end{array}\right)=\frac{\alpha(\alpha-1) \cdots(\alpha-\eta+1)}{\eta !} .
$$

In Appendix 1, we showed that the Eq. (4) is equivalent to the well-known expression $[30,31]$

$$
P_{\eta}(\cos \vartheta)=\frac{1}{2^{\eta}} \sum_{k=0}^{\left[\frac{\eta}{2}\right]}(-1)^{k} \frac{(2 \eta-2 k) !}{k !(\eta-k) !(\eta-2 k) !} \cos ^{\eta-2 k} \vartheta,
$$

where $\left[\frac{\eta}{2}\right]$ stands for the integer part of $\frac{\eta}{2}$. After substituting Eq. (2) into Eq. (4) and utilizing the trinomial expansion, the matrix elements, Eq. (3), read

$$
\begin{aligned}
V_{l_{1}, m_{1}, l_{2}, m_{2}}^{l_{1}^{\prime}, m_{1}^{\prime}, l_{2}^{\prime}, m_{2}^{\prime}}= & \sum_{\eta} V_{\eta}(R, r) 2^{\eta} \sum_{k=0}^{\eta}\left(\begin{array}{l}
\eta \\
k
\end{array}\right)\left(\begin{array}{c}
\frac{\eta+k-1}{2} \\
\eta
\end{array}\right) \sum_{\substack{k_{1}, k_{2}, k_{3} \geq 0 \\
k_{1}+k_{2}+k_{3}=k}}\left(\begin{array}{c}
k \\
k_{1}, k_{2}, k_{3}
\end{array}\right) \\
& \times \prod_{j=1}^{2} I\left(k_{3}, k_{1}+k_{2}, k_{1}, k_{2}, l_{j}^{\prime}, m_{j}^{\prime}, l_{j}, m_{j}\right),
\end{aligned}
$$

where $\left(\begin{array}{c}k \\ k_{1}, k_{2}, k_{3}\end{array}\right)=\frac{k !}{k_{1} ! k_{2} ! k_{3} !}$. This formula can be straight implemented in any quantum chemistry package or atomic and molecular physics program, provided that the analytical form of such a general integral is known

$$
\begin{aligned}
I\left(n_{1}, n_{2}, n_{3}, n_{4}, l_{j}^{\prime}, m_{j}^{\prime}, l_{j}, m_{j}\right)= & \int_{0}^{\pi} \int_{0}^{2 \pi} Y_{l_{j}^{\prime}, m_{j}^{\prime}}^{*}\left(\theta_{j}, \phi_{j}\right) \cos ^{n_{1}} \theta_{j} \sin ^{n_{2}} \theta_{j} \\
& \times \cos ^{n_{3}} \phi_{j} \sin ^{n_{4}} \phi_{j} Y_{l_{j}, m_{j}}\left(\theta_{j}, \phi_{j}\right) \sin \theta_{j} d \phi_{j} d \theta_{j}
\end{aligned}
$$

with $j=1,2$ and non-negative integers $n_{1}, n_{2}, n_{3}, n_{4}$. For simplicity, we henceforth omit the index $j$. We use mostly recommended and broadly accepted notation of the spherical harmonics, where the factor of $(-1)^{m}$, i.e., the Condon-Shortley phase, is included in the associated Legendre polynomial $\left(P_{l}^{m}\right)$, see Chapter 15.4 in Ref. [30]. Thus,

$$
Y_{l, m}=N_{l, m} P_{l}^{m}(\cos \theta) e^{i m \phi},
$$

where $l=0,1,2, \ldots,-l \leq m \leq l$, and $N_{l, m}$ is a normalization constant given by

$$
N_{l, m}=\sqrt{\frac{1}{2 \pi}} N_{l, m}^{\theta}=\sqrt{\frac{2 l+1}{4 \pi} \frac{(l-m) !}{(l+m) !}} .
$$

For negative $m$ values, the associated Legendre polynomial reads

$$
P_{l}^{-m}=(-1)^{m} \frac{(l-m) !}{(l+m) !} P_{l}^{m} .
$$


The integral $I\left(n_{1}, n_{2}, n_{3}, n_{4}, l^{\prime}, m^{\prime}, l, m\right)$, Eq. (8), can be factorized into two independent integrals:

$$
\begin{gathered}
I^{\theta}\left(n_{1}, n_{2}, l^{\prime}, m^{\prime}, l, m\right)=N_{l^{\prime}, m^{\prime}}^{\theta} N_{l, m}^{\theta} \int_{0}^{\pi} \cos ^{n_{1}} \theta \sin ^{n_{2}} \theta P_{l^{\prime}}^{m^{\prime}}(\cos \theta) P_{l}^{m}(\cos \theta) \sin \theta d \theta \\
I^{\phi}\left(n_{3}, n_{4}, m^{\prime}, m\right)=\frac{1}{2 \pi} \int_{0}^{2 \pi} \cos ^{n_{3}} \phi \sin ^{n_{4}} \phi e^{i\left(m-m^{\prime}\right) \phi} d \phi
\end{gathered}
$$

\subsection{Integral over $\theta$}

Substituting $\cos \theta$ by $x$ in Eq. (12) yields

$$
I^{\theta}\left(n_{1}, n_{2}, l^{\prime}, m^{\prime}, l, m\right)=N_{l^{\prime}, m^{\prime}}^{\theta} N_{l, m}^{\theta} \int_{-1}^{1} x^{n_{1}}\left(\sqrt{1-x^{2}}\right)^{n_{2}} P_{l^{\prime}}^{m^{\prime}}(x) P_{l}^{m}(x) d x .
$$

Based on the Schmied expression [32],

$$
x^{n}=\sum_{l=n, n-2, \ldots} \frac{n !(2 l+1)}{2^{\frac{n-l}{2}}\left(\frac{n-l}{2}\right) !(n+l+1) ! !} P_{l}(x), \quad n=0,1,2, \ldots,
$$

we expand the first term inside the integral $I^{\theta}$ by means of Legendre polynomials $P_{l}$. Note that $P_{l}(x)=P_{l}^{0}(x)$, therefore, further we use the form of the above equation with the associated Legendre polynomials. We also represent the second term in the integral $I^{\theta}$ by the associated Legendre polynomials utilizing

$$
P_{l}^{l}(x)=(-1)^{l}(2 l-1) ! !\left(\sqrt{1-x^{2}}\right)^{l} .
$$

Consequently, the considered integral (14) becomes

$$
\begin{aligned}
I^{\theta}\left(n_{1}, n_{2}, l^{\prime}, m^{\prime}, l, m\right)= & N_{l^{\prime}, m^{\prime}}^{\theta} N_{l, m}^{\theta} \frac{n_{1} !(-1)^{n_{2}}}{\left(2 n_{2}-1\right) ! !} \sum_{k_{1}=n_{1}, n_{1}-2, \ldots} \lambda\left(n_{1}, k_{1}\right) \\
& \times \int_{-1}^{1} P_{k_{1}}^{0}(x) P_{n_{2}}^{n_{2}}(x) P_{l^{\prime}}^{m^{\prime}}(x) P_{l}^{m}(x) d x
\end{aligned}
$$

where

$$
\lambda\left(n_{1}, k_{1}\right)=\frac{2 k_{1}+1}{2^{\frac{n_{1}-k_{1}}{2}}\left(\frac{n_{1}-k_{1}}{2}\right) !\left(n_{1}+k_{1}+1\right) ! !} .
$$

Dong and Lemus showed in Ref. [33] a general expression for the overlap integral of an arbitrary number of associated Legendre polynomials. Here, we present the proper formula adjusted to our problem of four associated Legendre polynomials, namely 


$$
\begin{aligned}
\int_{-1}^{1} P_{p_{1}}^{q_{1}}(x) P_{p_{2}}^{q_{2}}(x) P_{p_{3}}^{q_{3}}(x) P_{p_{4}}^{q_{4}}(x) d x= & \sqrt{\frac{\left(p_{1}+q_{1}\right) !\left(p_{2}+q_{2}\right) !\left(p_{3}+q_{3}\right) !\left(p_{4}+q_{4}\right) !}{\left(p_{1}-q_{1}\right) !\left(p_{2}-q_{2}\right) !\left(p_{3}-q_{3}\right) !\left(p_{4}-q_{4}\right) !}} \\
& \times \sum_{p_{12}} \sum_{p_{123} p_{1234}} G_{12} G_{123} G_{1234} \\
& \times \sqrt{\frac{\left(p_{1234}-q_{1234}\right) !}{\left(p_{1234}+q_{1234}\right) !}} \int_{-1}^{1} P_{p_{1234}}^{q_{1234}}(x) d x,
\end{aligned}
$$

where

$$
\begin{aligned}
& G_{12}=(-1)^{q_{12}}\left(2 p_{12}+1\right)\left(\begin{array}{ccc}
p_{1} & p_{2} & p_{12} \\
0 & 0 & 0
\end{array}\right)\left(\begin{array}{ccc}
p_{1} & p_{2} & p_{12} \\
q_{1} & q_{2} & -q_{12}
\end{array}\right), \\
& \left|p_{1}-p_{2}\right| \leq p_{12} \leq p_{1}+p_{2}, q_{12}=q_{1}+q_{2}, p_{12} \geq q_{12} \text {, } \\
& G_{123}=(-1)^{q_{123}}\left(2 p_{123}+1\right)\left(\begin{array}{ccc}
p_{12} & p_{3} & p_{123} \\
0 & 0 & 0
\end{array}\right)\left(\begin{array}{ccc}
p_{12} & p_{3} & p_{123} \\
q_{12} & q_{3} & -q_{123}
\end{array}\right), \\
& \left|p_{12}-p_{3}\right| \leq p_{123} \leq p_{12}+p_{3}, q_{123}=q_{1}+q_{2}+q_{3}, p_{123} \geq q_{123} \text {, } \\
& G_{1234}=(-1)^{q_{1234}}\left(2 p_{1234}+1\right)\left(\begin{array}{ccc}
p_{123} & p_{4} & p_{1234} \\
0 & 0 & 0
\end{array}\right)\left(\begin{array}{lll}
p_{123} & p_{4} & p_{1234} \\
q_{123} & q_{4} & -q_{1234}
\end{array}\right), \\
& \left|p_{123}-p_{4}\right| \leq p_{1234} \leq p_{123}+p_{4}, q_{1234}=q_{1}+q_{2}+q_{3}+q_{4}, p_{1234} \geq q_{1234} \text {, } \\
& \int_{-1}^{1} P_{p_{1234}}^{q_{123}}(x) d x=\left\{\begin{array}{cc}
2 & \text { if } p_{1234}=q_{1234}=0 \\
\mathcal{I}\left(p_{1234}, q_{1234}\right) & \text { otherwise }
\end{array},\right. \\
& \mathcal{I}\left(p_{1234}, q_{1234}\right)=\frac{\left[(-1)^{p_{1234}}+(-1)^{q_{1234}} 2^{q_{1234}-2} q_{1234} \Gamma\left(\frac{p_{1234}}{2}\right) \Gamma\left(\frac{p_{1234}+q_{1234}+1}{2}\right)\right.}{\Gamma\left(\frac{p_{1234}-q_{1234}+2}{2}\right) \Gamma\left(\frac{p_{1234}+3}{2}\right)} .
\end{aligned}
$$

In Eqs. (20)-(22), ( : : : ) denotes a Wigner $3 j$ symbol. It should be noted that the above formula for the overlap integral holds for all $q_{1}, q_{2}, q_{3}, q_{4} \geq 0$, while for negative values, one has to apply Eq. (11). Moreover, the integral is equal to zero when $p_{1}+p_{2}+p_{12}$ or $p_{12}+p_{3}+p_{123}$ or $p_{123}+p_{4}+p_{1234}$ is odd.

\subsection{Integral over $\phi$}

Let us now focus on the integral with respect to $\phi$, i.e., on Eq. (13). We represent the exponential function by means of the trigonometric ones using Euler's formula and perform its binomial expansion. Then, we obtain 


$$
\begin{aligned}
I^{\phi}\left(n_{3}, n_{4}, m^{\prime}, m\right)= & \frac{1}{2 \pi} \sum_{k=0}^{\left|m-m^{\prime}\right|} \delta^{k} i^{k}\left(\begin{array}{c}
\left|m-m^{\prime}\right| \\
k
\end{array}\right) \\
& \times \int_{0}^{2 \pi} \cos ^{\left|m-m^{\prime}\right|-k+n_{3}} \phi \sin ^{k+n_{4}} \phi d \phi,
\end{aligned}
$$

where

$$
\delta=\left\{\begin{array}{rll}
1 & \text { for } & m \geq m^{\prime} \\
-1 & \text { for } & m<m^{\prime}
\end{array}\right.
$$

and $i$ is the imaginary unit $\left(i^{2}=-1\right)$. As we showed in Appendix 2, the above internal integral may be written in the form of the beta function

$$
\begin{aligned}
\int_{0}^{2 \pi} \cos ^{\left|m-m^{\prime}\right|-k+n_{3}} \phi \sin ^{k+n_{4}} \phi d \phi= & \frac{1}{2} B\left(\frac{\left|m-m^{\prime}\right|-k+n_{3}+1}{2}, \frac{k+n_{4}+1}{2}\right) \\
& \times\left(1+(-1)^{\left|m-m^{\prime}\right|-k+n_{3}}\right) \\
& \times\left(1+(-1)^{\left|m-m^{\prime}\right|+n_{3}+n_{4}}\right) .
\end{aligned}
$$

Since there is a close relationship between the beta function and the gamma function, see Eq. (47) in Appendix 2, the integral over $\phi$ reads

$$
\begin{aligned}
I^{\phi}\left(n_{3}, n_{4}, m^{\prime}, m\right)= & \frac{\left(1+(-1)^{\left|m-m^{\prime}\right|+n_{3}+n_{4}}\right)}{4 \pi\left(\frac{\left|m-m^{\prime}\right|+n_{3}+n_{4}}{2}\right) !} \sum_{k=0}^{\left|m-m^{\prime}\right|} \delta^{k} i^{k}\left(\begin{array}{c}
\left|m-m^{\prime}\right| \\
k
\end{array}\right) \\
& \times \Gamma\left(\frac{\left|m-m^{\prime}\right|-k+n_{3}+1}{2}\right) \Gamma\left(\frac{k+n_{4}+1}{2}\right) \\
& \times\left(1+(-1)^{\left|m-m^{\prime}\right|-k+n_{3}}\right) .
\end{aligned}
$$

It is clear that this integral vanishes if $\left|m-m^{\prime}\right|+n_{3}+n_{4}$ is odd.

\section{Compact expression}

The matrix elements, Eq. (3), can be also found based on the theorem of addition of spherical harmonics [31, 34]

$$
P_{\eta}(\cos \vartheta)=\frac{4 \pi}{2 \eta+1} \sum_{m_{\eta}=-\eta}^{\eta} Y_{\eta, m_{\eta}}^{*}\left(\theta_{1}, \phi_{1}\right) Y_{\eta, m_{\eta}}\left(\theta_{2}, \phi_{2}\right)
$$

where $\vartheta$ is the angle between two directions described by $\left(\theta_{1}, \phi_{1}\right)$ and $\left(\theta_{2}, \phi_{2}\right)$, then

$$
V_{l_{1}, m_{1}, l_{2}, m_{2}}^{l_{1}^{\prime}, m_{1}^{\prime}, l_{2}^{\prime}, m_{2}^{\prime}}=\sum_{\eta} V_{\eta}(R, r) \frac{4 \pi}{2 \eta+1} \sum_{m_{\eta}=-\eta}^{\eta}\left\langle Y_{l_{1}^{\prime}, m_{1}^{\prime}} Y_{l_{2}^{\prime}, m_{2}^{\prime}}\left|Y_{\eta, m_{\eta}}^{*} Y_{\eta, m_{\eta}}\right| Y_{l_{1}, m_{1}} Y_{l_{2}, m_{2}}\right\rangle .
$$


The closed-form expression for the integral with three spherical harmonics is given by $[31,34]$

$$
\begin{aligned}
\int_{0}^{\pi} \int_{0}^{2 \pi} Y_{p_{1}, q_{1}} Y_{p_{2}, q_{2}} Y_{p_{3}, q_{3}} \sin \theta d \phi d \theta= & \sqrt{\frac{\left(2 p_{1}+1\right)\left(2 p_{2}+1\right)\left(2 p_{3}+1\right)}{4 \pi}} \\
& \times\left(\begin{array}{ccc}
p_{1} & p_{2} & p_{3} \\
0 & 0 & 0
\end{array}\right)\left(\begin{array}{lll}
p_{1} & p_{2} & p_{3} \\
q_{1} & q_{2} & q_{3}
\end{array}\right) .
\end{aligned}
$$

When we include $Y_{p, q}^{*}=(-1)^{q} Y_{p,-q}$, the matrix elements are as follows

$$
\begin{aligned}
V_{l_{1}, m_{1}, l_{2}, m_{2}}^{l_{1}^{\prime}, m_{1}^{\prime}, l_{2}^{\prime}, m_{2}^{\prime}}= & (-1)^{m_{1}^{\prime}+m_{2}^{\prime}} \sqrt{\left(2 l_{1}^{\prime}+1\right)\left(2 l_{1}+1\right)\left(2 l_{2}^{\prime}+1\right)\left(2 l_{2}+1\right)} \\
& \times \sum_{\eta} V_{\eta}(R, r)\left(\begin{array}{ccc}
l_{1}^{\prime} & \eta & l_{1} \\
0 & 0 & 0
\end{array}\right)\left(\begin{array}{ccc}
l_{2}^{\prime} & \eta & l_{2} \\
0 & 0 & 0
\end{array}\right) \\
& \times \sum_{m_{\eta}=-\eta}^{\eta}(-1)^{m_{\eta}}\left(\begin{array}{cccc}
l_{1}^{\prime} & \eta & l_{1} \\
-m_{1}^{\prime} & -m_{\eta} & m_{1}
\end{array}\right)\left(\begin{array}{ccc}
l_{2}^{\prime} & \eta & l_{2} \\
-m_{2}^{\prime} & m_{\eta} & m_{2}
\end{array}\right) .
\end{aligned}
$$

In order to validate our mathematical derivations, we implemented both approaches in MATLAB [35] and performed calculations for the same large set of various input parameters. We obtained identical results, confirming the correctness of the findings.

\section{Concluding remarks}

We have derived the exact analytical angular matrix elements of the potential for the interaction of an atom with a closed-shell diatomic molecule, expanded in Legendre polynomials, in a basis set of products of two spherical harmonics. We presented two approaches. The first concerns the calculation of the integral involving arbitrary powers of trigonometric functions. The obtained results within this approach are generic and may be useful not only in studies of anisotropic molecular collisions but also in other different fields of theoretical physics and chemistry. The second approach is much shorter; we demonstrated the expression in a compact form that can be of great interest to experimentalists due to its coding simplicity, especially when adiabatic variational theory is applied.

\section{Appendix 1}

We demonstrate that the following representation for Legendre polynomials

$$
P_{\eta}(x)=2^{\eta} \sum_{k=0}^{\eta}\left(\begin{array}{l}
\eta \\
k
\end{array}\right)\left(\begin{array}{l}
\alpha \\
\eta
\end{array}\right) x^{k},
$$

where $\alpha=\frac{\eta+k-1}{2}$, is an alternative to the more common one [30, 31] 


$$
P_{\eta}(x)=\frac{1}{2^{\eta}} \sum_{k=0}^{\left[\frac{\eta}{2}\right]}(-1)^{k} \frac{(2 \eta-2 k) !}{k !(\eta-k) !(\eta-2 k) !} x^{\eta-2 k}
$$

where $\left[\frac{\eta}{2}\right]$ stands for the integer part of $\frac{\eta}{2}$. The generalized binomial coefficient is defined in Eq. (5). Since

$$
\begin{aligned}
\left(\begin{array}{c}
\frac{\eta+k-1}{2} \\
\eta
\end{array}\right) & =\frac{\frac{\eta+k-1}{2}\left(\frac{\eta+k-1}{2}-1\right) \cdots\left(\frac{\eta+k-1}{2}-\eta+1\right)}{\eta !} \\
& =\frac{1}{\eta !} \prod_{r=0}^{\eta-1}\left(\frac{\eta+k-1}{2}-r\right)
\end{aligned}
$$

one can see that the expression vanishes if $\eta+k-1$ is even. In other words, when $\eta$ and $k$ have opposite parity, one of the terms in the product is always zero, and then the product is zero. On the other hand, based on

$$
\begin{aligned}
& \left(\frac{2 n-1}{2}\right) !=\left(-\frac{1}{2}\right) ! \frac{(2 n-1) ! !}{2^{n}} \\
& \left(\frac{2 n+1}{-2}\right) !=\left(-\frac{1}{2}\right) ! \frac{(-2)^{n}}{(2 n-1) ! !}
\end{aligned}
$$

where $n$ is non-negative integer, the generalized binomial coefficient can be written as follows

$$
\left(\begin{array}{c}
\frac{\eta+k-1}{2} \\
\eta
\end{array}\right)=\frac{\left(\frac{\eta+k-1}{2}\right) !}{\eta !\left(\frac{\eta+k-1}{2}-\eta\right) !}=2^{-\eta}(-1)^{\frac{\eta-k}{2}} \frac{(\eta+k-1) ! !(\eta-k-1) ! !}{\eta !}
$$

Let us now consider the case for even $\eta$ and $k$. To carry out the summation over nonzero terms, we take $\eta=2 \xi$ and $k=2 k^{\prime}$, then Eq. (33) is

$$
P_{2 \xi}(x)=2^{2 \xi} \sum_{k^{\prime}=0}^{\xi}\left(\begin{array}{c}
2 \xi \\
2 k^{\prime}
\end{array}\right) 2^{-2 \xi}(-1)^{\frac{2 \xi-2 k^{\prime}}{2}} \frac{\left(2 \xi+2 k^{\prime}-1\right) ! !\left(2 \xi-2 k^{\prime}-1\right) ! !}{(2 \xi) !} x^{2 k^{\prime}} .
$$

When we apply

$$
(2 n) !=(2 n-1) ! ! n ! 2^{n},
$$

we obtain

$$
P_{2 \xi}(x)=\frac{1}{2^{2 \xi}} \sum_{k^{\prime}=0}^{\xi}(-1)^{\xi-k^{\prime}} \frac{\left(2 \xi+2 k^{\prime}\right) !}{\left(2 k^{\prime}\right) !\left(\xi+k^{\prime}\right) !\left(\xi-k^{\prime}\right) !} x^{2 k^{\prime}} .
$$

The next step is to reverse the order of summation by taking $k^{\prime}=\xi-k^{\prime \prime}$, 


$$
P_{2 \xi}(x)=\frac{1}{2^{2 \xi}} \sum_{k^{\prime \prime}=0}^{\xi}(-1)^{k^{\prime \prime}} \frac{\left(4 \xi-2 k^{\prime \prime}\right) !}{k^{\prime \prime} !\left(2 \xi-k^{\prime \prime}\right) !\left(2 \xi-2 k^{\prime \prime}\right) !} x^{2 \xi-2 k^{\prime \prime}},
$$

and revert to a form involving $\eta$ by recalling that $\eta=2 \xi$, which ultimately leads to

$$
P_{\eta}(x)=\frac{1}{2^{\eta}} \sum_{k^{\prime \prime}=0}^{\frac{\eta}{2}}(-1)^{k^{\prime \prime}} \frac{\left(2 \eta-2 k^{\prime \prime}\right) !}{k^{\prime \prime} !\left(\eta-k^{\prime \prime}\right) !\left(\eta-2 k^{\prime \prime}\right) !} x^{\eta-2 k^{\prime \prime}} .
$$

In the second case, where $\eta$ and $k$ are odd, we proceed similarly but we take $\eta=2 \xi+1$ and $k=2 k^{\prime}+1$. Then, we obtain

$$
P_{\eta}(x)=\frac{1}{2^{\eta}} \sum_{k^{\prime \prime}=0}^{\left[\frac{\eta}{2}\right]}(-1)^{k^{\prime \prime}} \frac{\left(2 \eta-2 k^{\prime \prime}\right) !}{k^{\prime \prime} !\left(\eta-k^{\prime \prime}\right) !\left(\eta-2 k^{\prime \prime}\right) !} x^{\eta-2 k^{\prime \prime}} .
$$

When we write the upper limit of summation for the first case as $\left[\frac{\eta}{2}\right]$, it can be noted that Eqs. (43) and (44) are exactly the same and fully agree with Eq. (34).

\section{Appendix 2}

Here, we present how to evaluate the following type of integral (which appeared in Eq. (25))

$$
\int_{0}^{2 \pi} \cos ^{s} x \sin ^{t} x d x
$$

where $s \geq 0$ and $t \geq 0$. Let us take the beta function with real and positive arguments

$$
B(m, n)=\int_{0}^{1} x^{m-1}(1-x)^{n-1} d x
$$

which may be expressed by the gamma function

$$
B(m, n)=\frac{\Gamma(m) \Gamma(n)}{\Gamma(m+n)} .
$$

The latter equation implies that $B(m, n)=B(n, m)$. By inserting the substitution $x=\cos ^{2} u$ into Eq. (46), we get

$$
B(m, n)=2 \int_{0}^{\pi / 2} \cos ^{2 m-1} u \sin ^{2 n-1} u d u .
$$

When we replace $2 m-1$ by $s$ and $2 n-1$ by $t$, we have 


$$
\frac{1}{2} B\left(\frac{s+1}{2}, \frac{t+1}{2}\right)=\int_{0}^{\pi / 2} \cos ^{s} u \sin ^{t} u d u=I^{(1)} .
$$

This expression almost corresponds to the one we are interested in, i.e., Eq. (45). The only problem is the limit of integration. Note that

$$
\int_{0}^{\pi} \cos ^{s} u \sin ^{t} u d u=I^{(1)}+\int_{\pi / 2}^{\pi} \cos ^{s} u \sin ^{t} u d u=I^{(2)} .
$$

The next step is a change of variables: $u=v+\pi / 2$. Since $\cos (v+\pi / 2)=-\sin v$, $\sin (v+\pi / 2)=\cos v$, and remembering that $B\left(\frac{s+1}{2}, \frac{t+1}{2}\right)=B\left(\frac{t+1}{2}, \frac{s+1}{2}\right)$, we may write

$$
I^{(2)}=I^{(1)}+\int_{0}^{\pi / 2}(-\sin v)^{s} \cos ^{t} v d v=I^{(1)}+(-1)^{s} I^{(1)}=I^{(1)}\left(1+(-1)^{s}\right) .
$$

In a similar manner, we have

$$
\int_{0}^{2 \pi} \cos ^{s} v \sin ^{t} v d v=I^{(2)}+\int_{\pi}^{2 \pi} \cos ^{s} v \sin ^{t} v d v=I^{(3)}
$$

Again, we carry out a change of variables: $v=x+\pi$. By taking into account that $\cos (x+\pi)=-\cos x$ and $\sin (x+\pi)=-\sin x$, we receive

$$
\begin{aligned}
I^{(3)} & =I^{(2)}+\int_{0}^{\pi}(-\cos x)^{s}(-\sin x)^{t} d x=I^{(2)}+(-1)^{s+t} I^{(2)} \\
& =I^{(2)}\left(1+(-1)^{s+t}\right) .
\end{aligned}
$$

We collect intermediate solutions

$$
I^{(3)}=I^{(2)}\left(1+(-1)^{s+t}\right)=I^{(1)}\left(1+(-1)^{S}\right)\left(1+(-1)^{s+t}\right)
$$

and finally obtain

$$
\int_{0}^{2 \pi} \cos ^{s} x \sin ^{t} x d x=\frac{1}{2} B\left(\frac{s+1}{2}, \frac{t+1}{2}\right)\left(1+(-1)^{s}\right)\left(1+(-1)^{s+t}\right) .
$$

Acknowledgements We would like to thank Piotr Jankowski for helpful discussions. This research was financially supported by the National Science Centre, Poland (Grant No. 2016/23/D/ST4/00341).

\section{Declarations}

Conflict of interest The authors declare that they have no conflict of interest.

Open Access This article is licensed under a Creative Commons Attribution 4.0 International License, which permits use, sharing, adaptation, distribution and reproduction in any medium or format, as long as you give appropriate credit to the original author(s) and the source, provide a link to the Creative Commons licence, and indicate if changes were made. The images or other third party material in this article 
are included in the article's Creative Commons licence, unless indicated otherwise in a credit line to the material. If material is not included in the article's Creative Commons licence and your intended use is not permitted by statutory regulation or exceeds the permitted use, you will need to obtain permission directly from the copyright holder. To view a copy of this licence, visit http://creativecommons.org/licen ses/by/4.0/.

\section{References}

1. A.B. Henson, S. Gersten, Y. Shagam, J. Narevicius, E. Narevicius, Observation of resonances in Penning ionization reactions at sub-kelvin temperatures in merged beams. Science 338, 234-238 (2012). https://doi.org/10.1126/science.1229141

2. E. Lavert-Ofir, Y. Shagam, A.B. Henson, S. Gersten, J. Kłos, P.S. Żuchowski, J. Narevicius, E. Narevicius, Observation of the isotope effect in sub-kelvin reactions. Nat. Chem. 6, 332-335 (2014). https://doi.org/10.1038/nchem.1857

3. Y. Shagam, A. Klein, W. Skomorowski, R. Yun, V. Averbukh, C.P. Koch, E. Narevicius, Molecular hydrogen interacts more strongly when rotationally excited at low temperatures leading to faster reactions. Nat. Chem. 7, 921-926 (2015). https://doi.org/10.1038/nchem.2359

4. J.L. Bohn, A.M. Rey, J. Ye, Cold molecules: progress in quantum engineering of chemistry and quantum matter. Science 357, 1002-1010 (2017). https://doi.org/10.1126/science.aam6299

5. C.P. Koch, M. Lemeshko, D. Sugny, Quantum control of molecular rotation. Rev. Mod. Phys. 91, 035005 (2019). https://doi.org/10.1103/revmodphys.91.035005

6. Y. Liu, L. Luo, Molecular collisions: from near-cold to ultra-cold. Front. Phys. 16, 42300 (2021). https://doi.org/10.1007/s11467-020-1037-6

7. A. Klein, Y. Shagam, W. Skomorowski, P.S. Żuchowski, M. Pawlak, N. Moiseyev, M.C. Janssen, S.Y.T. van de Meerakker, A. van der Avoird, C.P. Koch, E. Narevicius, Directly probing anisotropy in atom-molecule collisions through quantum scattering resonances. Nat. Phys. 13, 35-38 (2017). https://doi.org/10.1038/nphys3904

8. S.L. Holmgren, M. Waldman, W. Klemperer, Internal dynamics of van der Waals complexes. I. Born-Oppenheimer separation of radial and angular motion. J. Chem. Phys. 67, 4414-4422 (1977). https://doi.org/10.1063/1.434572

9. J.M. Hutson, An introduction to the dynamics of van der Waals molecules. Adv. Mol. Vibrat. Coll. Dyn. 1A, 1-45 (1991)

10. M.-L. Dubernet, J.M. Hutson, Atom-molecule van der Waals complexes containing open-shell atoms. I. General theory and bending levels. J. Chem. Phys. 101, 1939-1958 (1994). https://doi. org/10.1063/1.467704

11. M. Pawlak, Y. Shagam, E. Narevicius, N. Moiseyev, Adiabatic theory for anisotropic cold molecule collisions. J. Chem. Phys. 143, 074114 (2015). https://doi.org/10.1063/1.4928690

12. R.D. Levine, Adiabatic approximation for nonreactive, subexcitation, molecular collisions. J. Chem. Phys. 49, 51-55 (1968). https://doi.org/10.1063/1.1669858

13. M.H. Alexander, S. Gregurick, P.J. Dagdigian, G.W. Lemire, M.J. McQuaid, R.C. Sausa, Potential energy surfaces for the interaction of $\mathrm{CH}\left(X^{2} \Pi, B^{2} \Sigma^{-}\right)$with $\mathrm{Ar}$ and an assignment of the stretch-bend levels of the $\operatorname{ArCH}(B)$ van der Waals molecule. J. Chem. Phys. 101, 4547-4560 (1994). https://doi.org/10.1063/1.467442

14. W. Klopper, M. Quack, M.A. Suhm, A new ab initio based six-dimensional semi-empirical pair interaction potential for HF. Chem. Phys. Lett. 261, 35-44 (1996). https://doi.org/10.1016/00092614(96)00901-3

15. C. Leforestier, F. Gatti, R.S. Fellers, R.J. Saykally, Determination of a flexible (12D) water dimer potential via direct inversion of spectroscopic data. J. Chem. Phys. 117, 8710-8722 (2002). https://doi.org/10.1063/1.1514977

16. H. Li, P.-N. Roy, R.J. Le Roy, An "adiabatic-hindered-rotor" treatment allows para- $\mathrm{H}_{2}$ to be treated as if it were spherical. J. Chem. Phys. 133, 104305 (2010). https://doi.org/10.1063/1. 3476465

17. T. Zeng, H. Li, R.J. Le Roy, P.-N. Roy, "Adiabatic-hindered-rotor" treatment of the parahydrogen-water complex. J. Chem. Phys. 135, 094304 (2011). https://doi.org/10.1063/1.3626840 
18. Y. Scribano, A. Faure, Note: On the inclusion of a diagonal Born-Oppenheimer correction in the reduced dimensional treatment of the $\mathrm{H}_{2} \mathrm{O}$-para- $\mathrm{H}_{2}$ complex. J. Chem. Phys. 146, 226102 (2017). https://doi.org/10.1063/1.4986074

19. D.C. Clary, Fast chemical reactions: theory challenges experiment. Annu. Rev. Phys. Chem. 41, 61-90 (1990). https://doi.org/10.1146/annurev.pc.41.100190.000425

20. Y. Scribano, A. Faure, D. Lauvergnat, Rotational excitation of $\mathrm{H}_{2} \mathrm{O}$ by para- $\mathrm{H}_{2}$ from an adiabatically reduced dimensional potential. J. Chem. Phys. 136, 094109 (2012). https://doi.org/10. 1063/1.3690881

21. T.V. Tscherbul, A.A. Buchachenko, Adiabatic channel capture theory applied to cold atom-molecule reactions: $\mathrm{Li}+\mathrm{CaH} \rightarrow \mathrm{LiH}+\mathrm{Ca}$ at $1 \mathrm{~K}$. New J. Phys. 17, 035010 (2015). https://doi.org/10.1088/13672630/17/3/035010

22. M. Pawlak, Y. Shagam, A. Klein, E. Narevicius, N. Moiseyev, Adiabatic variational theory for cold atom-molecule collisions: application to a metastable helium atom colliding with ortho- and parahydrogen molecules. J. Phys. Chem. A 121, 2194-2198 (2017). https://doi.org/10.1021/acs.jpca. $6 \mathrm{~b} 13038$

23. M. Pawlak, A. Ben-Asher, N. Moiseyev, Simple closed-form expression for Penning reaction rate coefficients for cold molecular collisions by non-Hermitian time-independent adiabatic scattering theory. J. Chem. Theory Comput. 14, 236-241 (2018). https://doi.org/10.1021/acs.jctc.7b01017

24. D. Bhattacharya, A. Ben-Asher, I. Haritan, M. Pawlak, A. Landau, N. Moiseyev, Polyatomic ab initio complex potential energy surfaces: illustration of ultracold collisions. J. Chem. Theory Comput. 13, 1682-1690 (2017). https://doi.org/10.1021/acs.jctc.7b00083

25. M. Pawlak, P.S. Żuchowski, N. Moiseyev, P. Jankowski, Evidence of nonrigidity effects in the description of low-energy anisotropic molecular collisions of hydrogen molecules with excited metastable helium atoms. J. Chem. Theory Comput. 16, 2450-2459 (2020). https://doi.org/10.1021/acs. jetc.0c00183

26. M. Pawlak, P.S. Żuchowski, P. Jankowski, Kinetic isotope effect in low-energy collisions between hydrogen isotopologues and metastable helium atoms: theoretical calculations including the vibrational excitation of the molecule. J. Chem. Theory Comput. 17, 1008-1016 (2021). https://doi.org/ 10.1021/acs.jctc.0c01122

27. D. Bhattacharya, M. Pawlak, A. Ben-Asher, A. Landau, I. Haritan, E. Narevicius, N. Moiseyev, Quantum effects in cold molecular collisions from spatial polarization of electronic wave function. J. Phys. Chem. Lett. 10, 855-863 (2019). https://doi.org/10.1021/acs.jpclett.8b03807

28. J.J. Spake, D.K. Sing, T.M. Evans, A. Oklopčić, V. Bourrier, L. Kreidberg, B.V. Rackham, J. Irwin, D. Ehrenreich, A. Wyttenbach, H.R. Wakeford, Y. Zhou, K.L. Chubb, N. Nikolov, J.M. Goyal, G.W. Henry, M.H. Williamson, S. Blumenthal, D.R. Anderson, C. Hellier, D. Charbonneau, S. Udry, N. Madhusudhan, Helium in the eroding atmosphere of an exoplanet. Nature 557, 68-70 (2018). https://doi.org/10.1038/s41586-018-0067-5

29. I.C. Percival, M.J. Seaton, The partial wave theory of electron-hydrogen atom collisions. Math. Proc. Camb. Philos. Soc. 53, 654-662 (1957). https://doi.org/10.1017/s0305004100032722

30. G.B. Arfken, H.J. Weber, F.E. Harris, Mathematical Methods for Physicists (7th edn., Academic Press, 2013)

31. A.R. Edmonds, Angular Momentum in Quantum Mechanics (Princeton University Press, Princeton, New Jersey, 1996)

32. E.W. Weisstein, From MathWorld - A Wolfram web resource. https://mathworld.wolfram.com/ LegendrePolynomial.html

33. S.-H. Dong, R. Lemus, The overlap integral of three associated Legendre polynomials. Appl. Math. Lett. 15, 541-546 (2002). https://doi.org/10.1016/s0893-9659(02)80004-0

34. I.I. Sobelman, Atomic Spectra and Radiative Transitions (Springer, Berlin, 1996)

35. The Mathworks, Inc., Natick, Massachusetts, MATLAB version 9.4.0.949201 (R2018a Update 6) (2018)

Publisher's Note Springer Nature remains neutral with regard to jurisdictional claims in published maps and institutional affiliations. 